\title{
A Country-Wide Study of Spoligotype and Drug Resistance Characteristics of Mycobacterium tuberculosis Isolates from Children in China
}

\author{
Weiwei Jiao $^{19}$, Zhiguang Liu',39, Rui Han ${ }^{19}$, Xiuqin Zhao ${ }^{2,3}$, Fang Dong ${ }^{1}$, Haiyan Dong ${ }^{2,3}$, \\ Hairong Huang ${ }^{4}$, Jianling Tian ${ }^{1}$, Qinjing Li ${ }^{1}$, Lulu Lian ${ }^{2,3}$, Qingqin Yin ${ }^{1}$, Wenqi Song ${ }^{1}$, Kanglin Wan ${ }^{2,3 *}$, A- \\ dong Shen ${ }^{1 *}$ \\ 1 Key Laboratory of Major Diseases in Children and National Key Discipline of Pediatrics (Capital Medical University), Ministry of Education, Beijing Pediatric Research \\ Institute, Beijing Children's Hospital, Capital Medical University, Beijing, China, 2 National Institute for Communicable Disease Control and Prevention, Chinese Center for \\ Disease Control and Prevention, State Key Laboratory for Infectious Disease Prevention and Control, Beijing, China, 3 Collaborative Innovation Center for Diagnosis and \\ Treatment of Infectious Diseases, Hangzhou, Zhejiang, China, 4 National Tuberculosis Clinical Laboratory, Beijing Chest Hospital, Capital Medical University, Beijing, China
}

\begin{abstract}
Background: Tuberculosis (TB) is still a big threat to human health, especially in children. However, an isolation of Mycobacterium tuberculosis culture from pediatric cases remains a challenge. In order to provide some scientific basis for children TB control, we investigated the genotyping and drug resistance characteristics of $M$. tuberculosis isolates from pediatric cases in China.

Methodology/Principal Findings: In this study, a total of 440 strains including 90 from children $(<15$ years), 159 from adolescents (15-18 years) and 191 from adults (>18 years) isolated in 25 provinces across China were subjected to spoligotyping and drug susceptibility testing. As a result, Beijing family strains were shown to remain predominant in China (85.6\%, $81.1 \%$ and $75.4 \%$ in three above groups, respectively), especially among new children cases $(91.0 \%$ vs. $69.6 \%$ in previously treated cases, $\mathrm{P}=0.03$ ). The prevalence of the Beijing genotype isolates was higher in northern and central China in the total collection $(85.1 \%$ in northern and $83.9 \%$ in central vs. $61.6 \%$ in southern China, $\mathrm{P}<0.001)$ and a similar trend was seen in all three age groups $(P=0.708,<0.001$ and 0.025 , respectively). In adolescents, the frequencies of isoniazid (INH)resistant and ethambutol (EMB)-resistant isolates were significantly higher among Beijing strains compared to non-Beijing genotype strains $(\mathrm{P}=0.028$ for $\mathrm{INH}$ and $\mathrm{P}=0.027$ for $\mathrm{EMB})$. Furthermore, strong association was observed between resistance to rifampicine (RIF), streptomycin (STR) and multidrug resistance (MDR) among Beijing compared to non-Beijing strains in previously treated cases of children $(P=0.01,0.01$ and 0.025 , respectively).
\end{abstract}

Conclusion/Significance: Beijing family was more prevalent in northern and central China compared to southern China and these strains were predominant in all age groups. The genetic diversity of $M$. tuberculosis isolates from children was similar to that found in adolescents and adults. Beijing genotype was associated with RIF, STR and MDR resistance in previously treated children.

Citation: Jiao W, Liu Z, Han R, Zhao X, Dong F, et al. (2013) A Country-Wide Study of Spoligotype and Drug Resistance Characteristics of Mycobacterium tuberculosis Isolates from Children in China. PLoS ONE 8(12): e84315. doi:10.1371/journal.pone.0084315

Editor: Igor Mokrousov, St. Petersburg Pasteur Institute, Russian Federation

Received November 1, 2013; Accepted November 21, 2013; Published December 30, 2013

Copyright: (๑) 2013 Jiao et al. This is an open-access article distributed under the terms of the Creative Commons Attribution License, which permits unrestricted use, distribution, and reproduction in any medium, provided the original author and source are credited.

Funding: This study was supported by the Capital Health Research and Development of Special Grant (No. 2011-1007-01, No. 2011-2009-05), National Science and Technology Major Project of China (2013ZX10003003), Open Project of Beijing Key Laboratory of Capital Medical University (2013NYJH02) and National Natural Science Foundation of China (No. 81271889). The funders had no role in study design, data collection and analysis, decision to publish, or preparation of the manuscript.

Competing Interests: The authors have declared that no competing interests exist.

*E-mail: shenad16@hotmail.com (AS); wankanglin@icdc.cn (KW).

9 These authors contributed equally to this work.

\section{Introduction}

Tuberculosis (TB), caused by Mycobacterium tuberculosis ( $M$. tuberculosis), still remains a major threat to human health. TB among children is especially important to public health workers since it is an indicator of recent transmission in the community. However, data on pediatric TB is limited and often underestimated because of diagnostic challenges. According to WHO tuberculosis report in 2012, there were estimated 0.5 million TB cases and 64,000 deaths among children in 2011 [1]. In the 37 countries providing multidrug-resistant tuberculosis [MDR-TB, characterized by resistance to at least isoniazid (INH) and rifampicin (RIF)] data including children, children represented $1-13 \%$ of total enrolments [1]. This was also the first time to include estimates of $\mathrm{TB}$ burden among children in the global tuberculosis report.

China is one of the high burden countries, ranking the second of total TB patients globally. However the most recent available data of $\mathrm{TB}$ in children came from the fourth national TB survey 
conducted in 2000. It was estimated that the prevalence of TB among 0-14 years old children was 9\%, and there were 266,000 children cases with active pulmonary $\mathrm{TB}$, making a serious threat to children health in China [2]. Therefore, more attention should be paid to $M$. tuberculosis strains isolated from children, which can provide the useful information for effective TB control.

The molecular typing methods have become the useful tool in the control of TB, which help to indicate possible epidemiological links between TB patients, detect the outbreaks and laboratory cross-contamination. Based on the DNA polymorphisms of $M$. tuberculosis genome, a number of genotyping methods have been developed and widely used in $M$. tuberculosis molecular typing. Spacer oligonucleotide typing (spoligotyping) is one of the easy-todo and standardized typing methods [3] although its discriminatory power is limited. However, spoligotyping has a continuing updated international spoligotype database [4], and also, it has long been considered the gold standard for identifying strains belonging to the Beijing family [5].

In this study, we investigated M. tuberculosis strains isolated from Chinese children (less than 15 years), adolescents (15-18 years) and adults (more than 18 years) using spoligotyping and drug susceptibility testing. The obtained results on genotypes and drug resistance in three age groups were compared to data on treatment status and patients' origin.

\section{Materials and Methods}

\section{Ethics Statement}

The research was approved by the Ethics Committee of Beijing Children's Hospital, Capital Medical University and National Institute for Communicable Disease Control and Prevention, Chinese Center for Disease Control and Prevention. The patients and parents/guardians on behalf of the children and adolescents included in this study were given an information consent form and they all signed to participate in the study.

\section{M. tuberculosis Isolates}

M. tuberculosis isolates were selected from the M. tuberculosis strain bank of Chinese Center for Disease Control and Prevention. The clinical strains in the bank were isolated from body fluid samples (sputum, bronchoalveolar lavage fluid, cerebrospinal fluid, pleural effusion, blood, or gastric juice) of the patients confirmed with TB in institutes for TB control and cure, as well as TB hospitals distributed in the following 25 provinces, municipalities and autonomous regions across China: Anhui, Beijing, Chongqing, Fujian, Gansu, Guangxi, Guizhou, Hebei, Henan, Hunan, Hubei, Heilongjiang, Inner Mongolia, Jilin, Jiangsu, Jiangxi, Liaoning, Qinghai, Shandong, Shaanxi, Shanxi, Sichuan, Tianjin, Xinjiang, and Xizang (Tibet). All the strains in the bank from patients less than 18 years old were included in this study, and the other group of strains from patients more than 18 years old was selected using random number table matched by region and isolation time. The basic information of eligible patients was also collected. Patients with new cases were those who had tuberculosis that had never been treated with anti-tuberculosis drugs or that had been treated for less than 1 month. Patients with previously treated cases were those who had been treated for tuberculosis for 1 month or longer.

\section{Drug Susceptibility Test}

The chosen strains were recovered on Loewenstein-Jensen medium for 4 weeks at $37^{\circ} \mathrm{C}$. Drug susceptibility testing to four first-line anti-TB drugs [INH, RIF, ethambutol (EMB) and streptomycin (STR)] was performed by proportion method as recommended by WHO [6]. The concentrations of different drugs in the media were as following: INH $0.2 \mu \mathrm{g} / \mathrm{mL}$, RIF $40 \mu \mathrm{g} / \mathrm{mL}$, EMB $2 \mu \mathrm{g} / \mathrm{mL}$, and STR $4 \mu \mathrm{g} / \mathrm{mL}$. The strain was considered resistant to specific drug when the growth rate was more than $1 \%$ compared to the control.

\section{Spoligotyping}

Total chromosomal DNA of strains was isolated following recommended method described by van Embden et al. [7]. Spoligotyping was performed as described by Kamerbeek et al. [3]. In short, the PCR amplified biotin-labeled DR locus was hybridized against membrane with immobilized 43 different DR spacers using Miniblotter MN45 apparatus (Immunetics, Cambridge, MA, USA). Resulting hybridization signals were revealed by chemiluminescence and visualized as profiles of discrete dots. The spoligoprofiles were entered into Excel spreadsheets and compared with SITVITWEB international spoligotype database [4].

\section{Statistical Analysis}

Tests for association between various factors (geographical distribution, drug resistance, new cases or previously treated cases) and genotypes were performed using the chi-square test with IBM SPSS Statistics 19. The level of significance was set at $\mathrm{P}<0.05$.

\section{Results}

\section{Study Population}

A total of 440 isolates were selected for this study, including 90 from children, 159 from adolescents, and 191 from adults. The male patients were prevalent in all three age groups and constituted 55.6\% (50/90), 52.2\% (83/159) and 63.9\% (122/ 191), respectively. The average age of patients in each group was 7.3 years, 16.9 years and 40.8 years. Newly diagnosed cases constitute $308(70.0 \%)$ individuals in our collection, and accounted for $74.4 \%$ (67/90), 69.8\% (111/159) and 68.1\% (130/191) among patients from children, adolescents and adults, respectively. All the patients enrolled in this study were proven to be unlinked based on conventional epidemiological investigation.

\section{Distribution of Spoligotypes in Different Groups}

In the 440 typed isolates, a total of 66 spoligotypes were identified (Table 1). Of them, 47 spoligotypes were present in the SITVITWEB database, while other 15 spoligotypes were not found in SITVITWEB and were named as New 1 to New 15 (Table 1). Family assignment revealed that 350 isolates $(79.5 \%)$ belonged to the Beijing family, followed by ill-defined $\mathrm{T}$ superfamily $(12.7 \%, 56 / 440)$ and Haarlem family $(2.0 \%, 9 / 440)$. In addition, strains from other families, such as CAS1-Delhi family, LAM9 family, S family, Manu_ancestor family, MANU2 family, were also found in this study although at very low rate ( $0.2 \%$ for all families except for $0.4 \%$ for LAM9 family).

Among 90 strains isolated from children, the Beijing family was predominant $(85.6 \%, 77 / 90)$, followed by $\mathrm{T}$ family $(8.9 \%, 8 / 90)$ and LAM family $(1.1 \%, 1 / 90)$ (Table 1). According to the SITVITWEB database, 87 pediatric isolates were assigned to the known SITs, while three isolates presented new patterns (New3, New12 and New13). Seventy-one (78.9\%) isolates showed a profile typical of the Beijing genotype (SIT1), and six had abridged Beijing-like spoligoprofiles (SIT190, SIT269, SIT621, SIT2413). The remaining 13 isolates were subdivided into 10 genotypes. The isolates from adolescents and adults were divided into five and seven clusters, respectively. The most prevalent family in the two groups was also the Beijing family $(81.1 \%$ in adolescents, $75.4 \%$ in adults), with $\mathrm{T}$ family $(10.7 \%$ in adolescents, $16.2 \%$ in adults) 


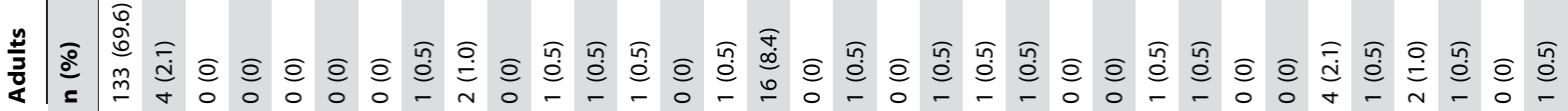

苞

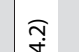

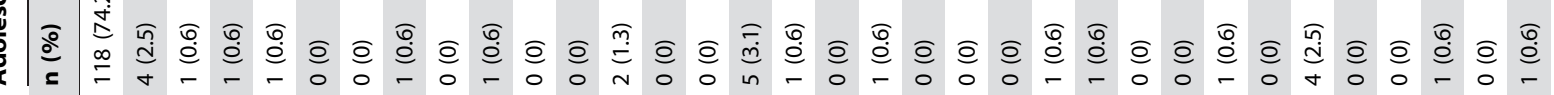

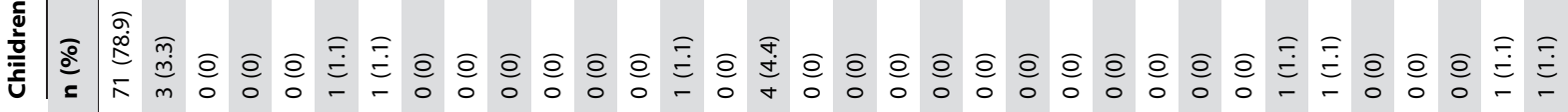

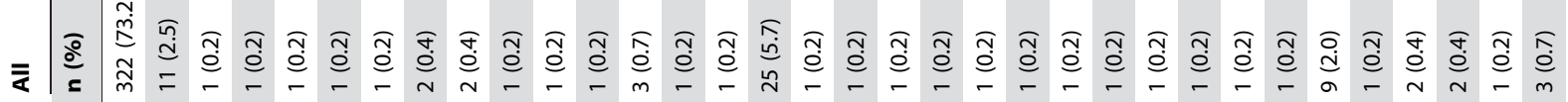

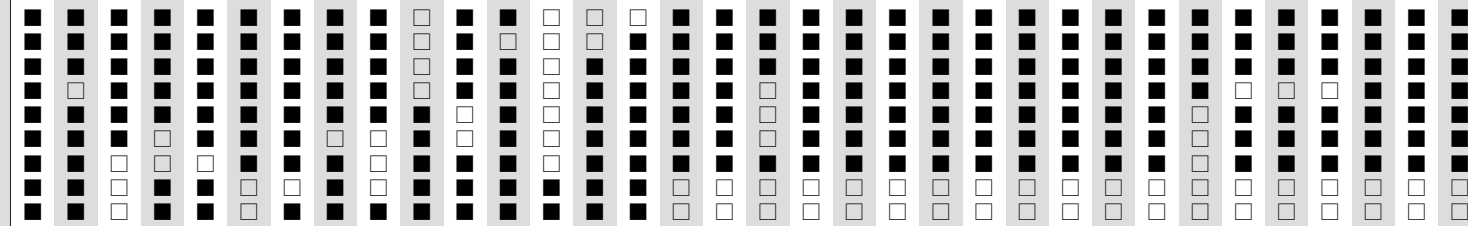

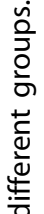

.$\subseteq$

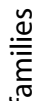

尝

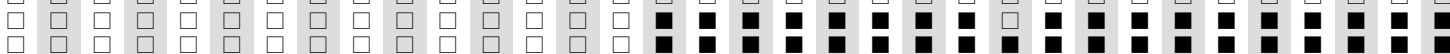

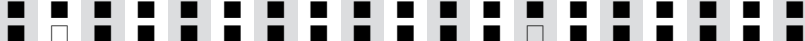

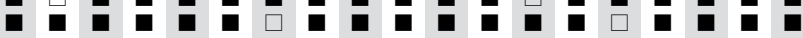

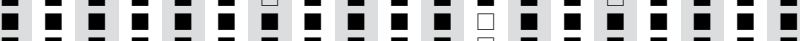

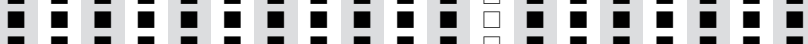

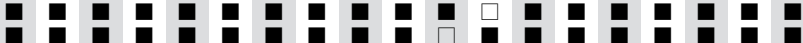

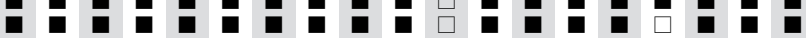

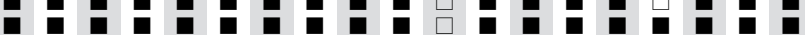

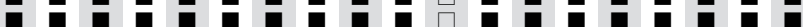

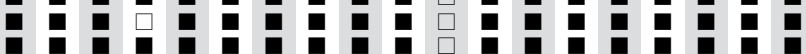

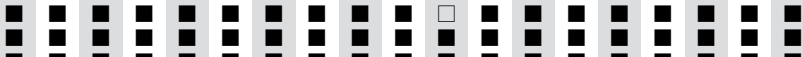

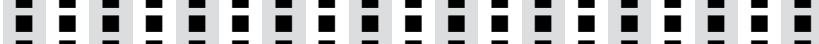

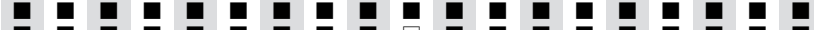

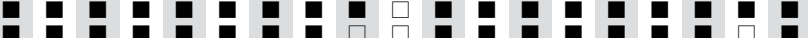

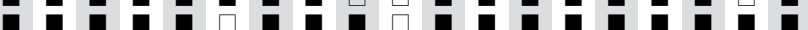

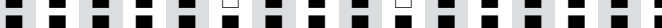

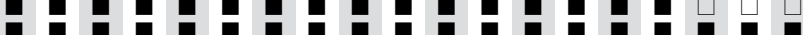

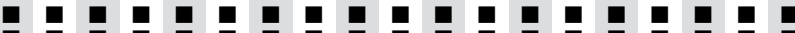

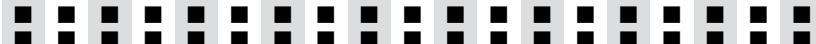

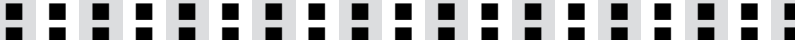

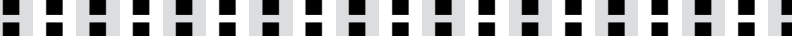

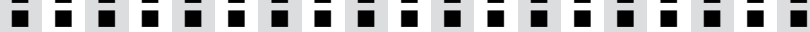

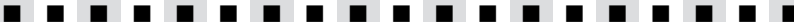

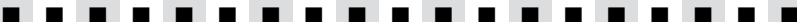

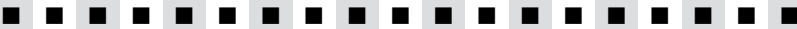

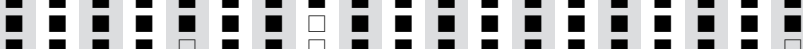

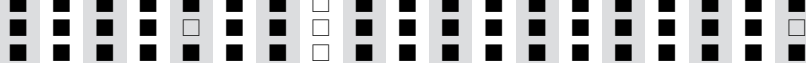

$\frac{\overline{0}}{\frac{0}{0}}$

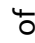

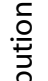

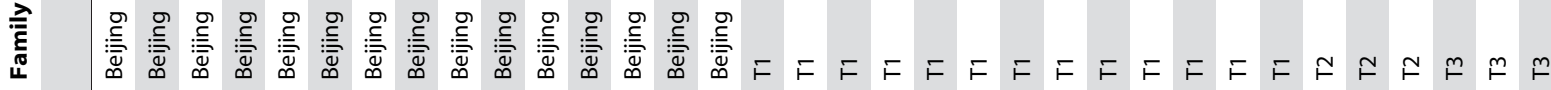
$\dot{-}$

卢 _. 

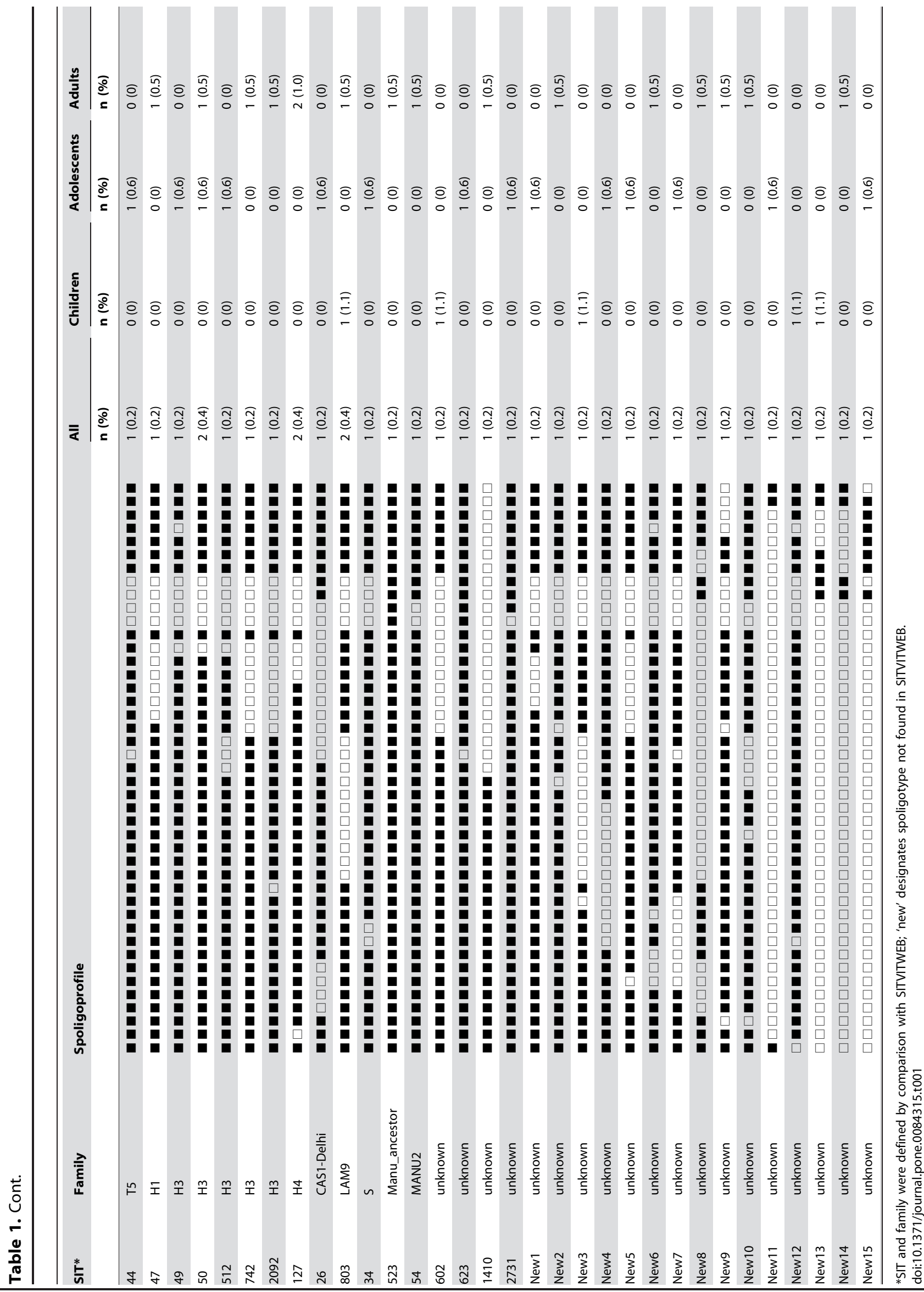
Table 2. Geographical distribution of Beijing family strains in different age groups.

\begin{tabular}{|c|c|c|c|c|c|c|c|}
\hline & \multicolumn{2}{|l|}{ Northern } & \multicolumn{2}{|l|}{ Central } & \multicolumn{2}{|l|}{ Southern } & \multirow{2}{*}{$P$ value } \\
\hline & Beijing, $n(\%)$ & non-Beijing, n(\%) & Beijing, $n(\%)$ & non-Beijing, n(\%) & Beijing, $n(\%)$ & non-Beijing, n(\%) & \\
\hline Children group $(n=90)$ & $55(87.3)$ & $8(12.7)$ & $15(83.3)$ & $3(16.7)$ & $7(77.8)$ & $2(22.2)$ & 0.708 \\
\hline Adolescent group $(n=159)$ & $70(89.7)$ & $8(10.3)$ & $30(90.9)$ & $3(9.1)$ & $29(60.4)$ & 19 (39.6) & $<0.001$ \\
\hline Adult group $(n=191)$ & $86(80.4)$ & $21(19.6)$ & $33(78.6)$ & $9(21.4)$ & $25(59.5)$ & $17(40.5)$ & 0.025 \\
\hline Total $(n=440)$ & $211(85.1)$ & 37 (14.9) & 78 (83.9) & $15(16.1)$ & $61(61.6)$ & $38(38.4)$ & $<0.001$ \\
\hline
\end{tabular}

Note: Northern China: Beijing, Gansu, Hebei, Heilongjiang, Jilin, Liaoning, Inner Mongolia, Qinghai, Shanxi, Tianjin, Xizang (Tibet); Central China: Anhui, He'nan, Hubei, Jiangsu, Shangdong, Shanxi, Sichuan, Chongqing; Southern China: Fujian, Guangxi, Guizhou, Hu'nan, Jiangxi, Xinjiang.

doi:10.1371/journal.pone.0084315.t002

ranking the second (Table 1). There was no significant difference in the distribution of the Beijing family strains among the three age groups $(\mathrm{P}=0.123)$.

\section{Geographical Distribution of Beijing Family Strains in Different Age Groups}

According to the geographical division suggested by Wan et al. [8], the region of origin of strains were defined as (i) northern China (to the north of Yellow River, and Xizang), (ii) central China (between Yellow River and Yangtze River) and (iii) southern China (to the south of Yangtze River, and Xinjiang). As shown in Table 2, the highest density of the Beijing strains was observed in the northern and central part of China $(85.1 \%$ and $83.9 \%$, respectively), and the lowest was observed in the southern China $(61.6 \%)$. The difference was strongly significant $(\mathrm{P}<0.001)$. A similar trend of geographical distribution of the Beijing strains was seen in all three age groups, children, adolescents and adults. However, the statistically significant differences among northern, central and southern China were only found for adolescents and adults $(\mathrm{P}<0.001$ and 0.025 , respectively $)$, but not children $(\mathrm{P}=0.708)$. In addition, in southern China, the prevalence of the Beijing family strains in children was relatively higher than that in adolescents and adults (77.8\% vs. $60.4 \%$ and $59.5 \%)$.

\section{Distribution of the Beijing Family Strains in New and Previously Treated Cases}

Table 3 shows the distribution of the Beijing family strains in new and previously treated cases in three age groups. Among 308 new cases of the total sample, the prevalence rate of the Beijing family was almost the same among newly-diagnosed (79.9\%, 246/ $308)$ and previously treated cases $(78.8 \%, 104 / 132)$. After stratification by age, Beijing strains were found to be much more prevalent among new cases in children $(91.0 \%$ vs. $69.6 \%$ in the previously treated cases, $\mathrm{P}=0.029)$. In contrast, the proportion of the Beijing family strains was similar between new and previously treated cases in the adolescents and adults (Table 3).

\section{Comparison of the Beijing Genotype Strains with Drug Resistance}

The relationship between the Beijing genotype strains and resistance to anti-TB drugs was analyzed (Table 4). The rates of resistance to the first-line drugs and MDR in the Beijing family strains were higher than those in the non-Beijing strains in the total collection while this difference was statistically significant for EMB resistance $(P=0.009)$. After stratification by age, the drug resistance rates (all tested drugs and MDR) in the Beijing strains were still higher than those in the non-Beijing strains in all three groups, with the exception of RIF and INH resistance in adolescents $(34.0 \%$ vs. $38.3 \%$ for RIF, $43.8 \%$ vs. $46.8 \%$ for $\mathrm{INH}$ in the Beijing and non-Beijing strains, respectively). In addition, Beijing family strains were also found to be associated with $\mathrm{INH}(\mathrm{P}=0.028)$ and $\mathrm{EMB}(\mathrm{P}=0.027)$ resistance in adolescent group.

The relationship between Beijing genotype strains and first-line anti-TB drug resistance in new cases and previously treated cases was further analyzed, as shown in Table 5. The distribution of drug resistance between Beijing and non-Beijing strains among new cases and previously treated cases in the total collection was similar, with an exception for EMB resistance in previously treated cases $(47.1 \%$ in Beijing strains vs. $17.9 \%$ in non-Beijing strains, $\mathrm{P}=0.009)$. When the patients were divided into three age groups, no significant difference was noticed in adolescents and adults. However, in the previously treated cases in the children group, the resistance rate to all four first-line drugs and MDR was higher in Beijing genotype strains compared to strains of other genotypes $(81.3 \%$ vs. $14.3 \%$ for RIF, $75.0 \%$ vs. $28.6 \%$ for INH, $81.3 \%$ vs. $14.3 \%$ for SM, $50.0 \%$ vs. $14.3 \%$ for $\mathrm{EMB}, 75.0 \%$ vs. $14.3 \%$ for

Table 3. Distribution of Beijing genotype strains in new cases and previously treated cases in different age groups.

\begin{tabular}{|c|c|c|c|c|c|c|c|c|c|c|c|c|}
\hline & \multicolumn{2}{|l|}{ Children } & \multirow{3}{*}{$P$ value } & \multicolumn{2}{|c|}{ Adolescents } & \multirow{3}{*}{ P value } & \multicolumn{2}{|l|}{ Adults } & \multirow{2}{*}{$P$ value } & \multicolumn{2}{|l|}{ Total } & \multirow{2}{*}{$P$ value } \\
\hline & Beijing & $\begin{array}{l}\text { non- } \\
\text { Beijing }\end{array}$ & & Beijing & $\begin{array}{l}\text { non- } \\
\text { Beijing }\end{array}$ & & Beijing & $\begin{array}{l}\text { non- } \\
\text { Beijing }\end{array}$ & & Beijing & $\begin{array}{l}\text { non- } \\
\text { Beijing }\end{array}$ & \\
\hline & N (\%) & N (\%) & & N (\%) & N (\%) & & N (\%) & N (\%) & & N (\%) & N (\%) & \\
\hline New cases & $61(91.0)$ & $6(9.0)$ & 0.029 & $87(78.4)$ & 24 (21.6) & 0.195 & $98(75.4)$ & $32(24.6)$ & 1.000 & $246(79.9)$ & $62(20.1)$ & 0.897 \\
\hline $\begin{array}{l}\text { Previously } \\
\text { treated cases }\end{array}$ & $16(69.6)$ & $7(30.4)$ & & $42(87.5)$ & $6(12.5)$ & & $46(75.4)$ & $15(24.6)$ & & $104(78.8)$ & $28(21.2)$ & \\
\hline
\end{tabular}


Table 4. Drug resistance properties of the Beijing family strains and strains of other genotypes.

\begin{tabular}{|c|c|c|c|c|c|c|c|c|c|c|c|c|}
\hline & \multicolumn{2}{|l|}{ Children } & \multirow[t]{2}{*}{ P value } & \multicolumn{2}{|c|}{ Adolescents } & \multirow[t]{2}{*}{ P value } & \multicolumn{2}{|l|}{ Adults } & \multirow[t]{2}{*}{$P$ value } & \multicolumn{2}{|l|}{ Total } & \multirow[t]{2}{*}{$P$ value } \\
\hline & Beijing & $\begin{array}{l}\text { non- } \\
\text { Beijing }\end{array}$ & & Beijing & $\begin{array}{l}\text { non- } \\
\text { Beijing }\end{array}$ & & Beijing & $\begin{array}{l}\text { non- } \\
\text { Beijing }\end{array}$ & & Beijing & $\begin{array}{l}\text { non- } \\
\text { Beijing }\end{array}$ & \\
\hline & N (\%) & N (\%) & & N (\%) & N (\%) & & N (\%) & N (\%) & & N (\%) & N (\%) & \\
\hline RIF & $28(36.4)$ & $2(15.4)$ & 0.244 & $34(26.4)$ & $3(10.0)$ & 0.091 & 49 (34.0) & 18 (38.3) & 0.602 & $111(31.7)$ & $23(25.6)$ & 0.305 \\
\hline INH & $26(33.8)$ & $3(23.1)$ & 0.658 & $44(34.1)$ & $4(13.3)$ & 0.028 & $63(43.8)$ & $22(46.8)$ & 0.738 & $133(38.0)$ & $29(32.2)$ & 0.329 \\
\hline STR & 32 (41.6) & $4(30.8)$ & 0.551 & $39(30.2)$ & $9(30.0)$ & 1 & 64 (44.4) & 19 (40.4) & 0.735 & 135 (38.6) & 32 (35.6) & 0.628 \\
\hline EMB & $17(22.1)$ & $2(15.4)$ & 0.857 & $34(26.4)$ & $2(6.7)$ & 0.027 & 34 (23.6) & $6(12.8)$ & 0.148 & $85(24.3)$ & $10(11.1)$ & 0.009 \\
\hline MDR & $20(26.0)$ & $2(15.4)$ & 0.636 & $32(24.8)$ & $3(10.0)$ & 0.09 & $45(31.3)$ & 14 (29.8) & 0.859 & $97(27.7)$ & $19(21.1)$ & 0.229 \\
\hline
\end{tabular}

doi:10.1371/journal.pone.0084315.t004

MDR), and the difference was significant for RIF, SM and MDR $(\mathrm{P}=0.01,0.01$ and 0.025 , respectively).

\section{Discussion}

The present study aimed to investigate the genetic diversity and drug resistance of $M$. tuberculosis strains isolated from children TB patients and compared with adolescent and adult TB patients coming from different regions across China. Although these strains might be not representative of all strains present in the country, they provide a preliminary insight into the population structure of M. tuberculosis circulating in these specific groups, especially in view of the total lack of this kind of data on Chinese pediatric TB population. Our study found that the Beijing family strains were prevalent in the total collection $(79.5 \%)$. This result is similar to the two previous large-scale studies conducted in China $[9,10]$. Dong et al. analyzed 2,346 M. tuberculosis isolates from 13 provinces and showed that Beijing family isolates were prevalent all over the country $(74.08 \%)$ [9], while Guo et al. reported that $77.8 \%$ of 158 strains isolated in five provinces belonged to the Beijing genotype [10]. However, this rate was much lower $(62.2 \%)$ in a recent study of 4017 isolates from the National Drug Resistance Base-Line Surveillance of Tuberculosis [11]. The explanation of the difference may be that the strains used in the national survey were mainly from the southern China (61.3\%), where the Beijing strains showed a lower percentage (discussed below).

Here, the prevalence of the Beijing strains varied in different age groups, ranging from $85.56 \%$ in children, $81.13 \%$ in adolescents, to $75.39 \%$ in adults. Further comparison of the distribution of the Beijing strains between new cases and previously treated cases demonstrated that Beijing strains were significantly associated with new cases in children than in previously treated patients. It is known that the Beijing family strains are worldwide spread clones, some of which represent epidemic clones in some areas [12]. This particular genotype was for the first time identified in strains isolated in the Beijing area in China and thus named accordingly [13]. It was speculated that long-term Mycobacterium bovis BCG vaccination may be one of the selective forces implicated in the successful spread of the Beijing genotype at least in East Asia, although the association remains inconclusive [14-17]. Our results provide some evidence in support of the hypothesis that Beijing genotype may possess mechanisms to circumvent a BCG-induced immunity. Firstly, BCG was included in the planned vaccination program in China since mid-1970s, and the vaccination rates had

Table 5. Drug resistance properties of the Beijing family strains and strains of other genotypes in subgroups of new and previously treated cases.

\begin{tabular}{|c|c|c|c|c|c|c|c|c|c|c|c|c|c|}
\hline & & \multicolumn{2}{|l|}{ Children } & \multirow[t]{3}{*}{$P$ value } & \multicolumn{2}{|c|}{ Adolescent } & \multirow[t]{3}{*}{ P value } & \multicolumn{2}{|l|}{ Adult } & \multirow[t]{3}{*}{ P value } & \multicolumn{2}{|l|}{ Total } & \multirow[t]{2}{*}{$P$ value } \\
\hline & & Beijing & $\begin{array}{l}\text { non- } \\
\text { Beijing }\end{array}$ & & Beijing & $\begin{array}{l}\text { non- } \\
\text { Beijing }\end{array}$ & & Beijing & $\begin{array}{l}\text { non- } \\
\text { Beijing }\end{array}$ & & Beijing & $\begin{array}{l}\text { non- } \\
\text { Beijing }\end{array}$ & \\
\hline & & N (\%) & N (\%) & & N (\%) & N (\%) & & N (\%) & N (\%) & & N (\%) & N (\%) & \\
\hline \multirow[t]{5}{*}{ New cases } & RIF & $15(24.6)$ & $1(16.7)$ & 1.000 & $11(12.6)$ & $0(0)$ & 0.147 & $21(21.4)$ & $10(31.3)$ & 0.339 & $47(19.1)$ & $11(17.7)$ & 0.858 \\
\hline & $\mathrm{INH}$ & $14(23.0)$ & $1(16.7)$ & 1.000 & $20(23.0)$ & $1(4.2)$ & 0.073 & 32 (32.7) & $10(31.3)$ & 1 & $66(26.8)$ & $12(19.4)$ & 0.256 \\
\hline & STR & $19(31.1)$ & $3(50.0)$ & 0.629 & $18(20.7)$ & $6(25.0)$ & 0.780 & $37(37.8)$ & $9(28.1)$ & 0.397 & $74(30.1)$ & $18(29.0)$ & 0.879 \\
\hline & EMB & $9(14.8)$ & 1 (16.7) & 1.000 & $14(16.1)$ & $1(4.2)$ & 0.240 & $13(13.3)$ & $3(9.4)$ & 0.786 & $36(14.6)$ & $5(8.1)$ & 0.212 \\
\hline & MDR & $8(13.1)$ & $1(16.7)$ & 1.000 & $10(11.5)$ & $0(0)$ & 0.181 & $20(20.4)$ & 7 (21.9) & 1 & $38(15.4)$ & $8(12.9)$ & 0.694 \\
\hline \multirow{5}{*}{$\begin{array}{l}\text { Previously } \\
\text { treated cases }\end{array}$} & RIF & $13(81.3)$ & $1(14.3)$ & 0.010 & $23(54.8)$ & $3(50.0)$ & 1.000 & $28(60.9)$ & $8(53.3)$ & 0.764 & $64(61.5)$ & $12(42.9)$ & 0.088 \\
\hline & $\mathrm{INH}$ & $12(75.0)$ & $2(28.6)$ & 0.102 & $24(57.1)$ & $3(50.0)$ & 1.000 & $31(67.4)$ & $12(80.0)$ & 0.546 & $67(64.4)$ & $17(60.7)$ & 0.825 \\
\hline & STR & $13(81.3)$ & $1(14.3)$ & 0.010 & $21(50.0)$ & $3(50.0)$ & 1.000 & 27 (58.7) & $10(66.7)$ & 0.763 & $61(58.7)$ & $14(50.0)$ & 0.520 \\
\hline & EMB & $8(50.0)$ & $1(14.3)$ & 0.250 & $20(47.6)$ & 1 (16.7) & 0.322 & $21(45.7)$ & $3(20.0)$ & 0.127 & $49(47.1)$ & 5 (17.9) & 0.009 \\
\hline & MDR & $12(75.0)$ & 1 (14.3) & 0.025 & $22(52.4)$ & $3(50.0)$ & 1.000 & $25(54.3)$ & 7 (46.7) & 0.767 & $59(56.7)$ & $11(39.3)$ & 0.135 \\
\hline
\end{tabular}


already exceeded $85 \%$ after 1990 . Although the BCG vaccination information was not available in our study, we can assume that the vaccination rate in children and adolescents is higher than in adults. Therefore, the Beijing family strains might be more prevalent among younger population. Secondly, our data showed that Beijing strains were significantly associated with new cases than in previously treated patients in children. The selection of strains in the previously treated cases mainly comes from the use of anti-TB drugs. However, the newly diagnosed patients did not receive any or very short (less than 1 month) drug therapy. In this sense, the significantly higher proportion of Beijing strains in new cases of children might be explained by other factors, such as higher transmission ability under the pressure of BCG vaccination.

Analysis of the geographical distribution of the Beijing family strains showed similar general trends in all three age groups: the prevalence rate of the Beijing genotype was higher in northern and central China, and lower in southern China. This result is in agreement with a previous study showing that the prevalence of the Beijing genotype exhibited geographical variation from $54.50 \%$ to $92.59 \%$ across China whereas the highest prevalence was found in northern China, followed by central and southern China [9]. Another study focused on genotypic characteristics of childhood tuberculosis in Chongqing, southern China, found that Beijing lineages accounted for $64.8 \%$ of the all strains [18]. The proportion is much lower than in our study, but is in concordance with the previous surveillance in the surrounding areas of Chongqing, such as Sichuan province $(57.89 \%)$ and Hunan province $(66.00 \%)$ [9]. Thus a difference between our countrywide study and Chongqing pediatric study may be explained by geographical variation. Pang et al. suggested that the geographical distribution difference of the Beijing genotype strains in China might be caused by the different economic status and climate condition [11]. In addition, compared with adolescents and adults, the prevalence of the Beijing family strains in southern China was relatively high in children. This may be due to the small size of the isolates from south China in children, and the further study including larger samples should be undertaken.

It was reported that Beijing family strains were associated with drug resistance, which might drive the spread of this particular genotype. However, the relationship between the Beijing family strains and drug resistance varied in different studies [11,19-21]. A comprehensive review on the association of drug resistance with Beijing strains revealed that Beijing genotype was significantly associated with resistance in Denmark (RIF and EMB), Finland (RIF and STR), the Netherlands (STR), and Russia (all first-line drugs and MDR), while no association was found in most of the Asian studies [19]. Recently, a study analyzing 1,375 M. tuberculosis strains from six provinces in China revealed that Beijing strains were not associated with RIF, INH and MDR resistance [20], which was consistent with the above mentioned review [19] However, Pang and his colleagues using strains from national survey revealed that Beijing family strains exhibited a greater

\section{References}

1. WHO | Global tuberculosis report 2012. Available: http://www.who.int/tb/ publications/global_report/en/. Accessed 26 Aug 2013.

2. Li L, Duanmu H-J (2004) [The epidemic of childhood tuberculosis in China]. Zhonghua Yi Xue Za Zhi 84: 1678-1680.

3. Kamerbeek J, Schouls L, Kolk A, van Agterveld M, van Soolingen D, et al. (1997) Simultaneous detection and strain differentiation of Mycobacterium tuberculosis for diagnosis and epidemiology. J Clin Microbiol 35: 907-914.

4. Demay C, Liens B, Burguière T, Hill V, Couvin D, et al. (2012) SITVITWEB-a publicly available international multimarker database for studying Mycobacterium tuberculosis genetic diversity and molecular epidemiology. Infect Genet Evol 12: 755-766. association with resistance to RIF, INH, EMB, OFL and MDR than other $M$. tuberculosis spoligotypes [11]. Recently, a metaanalysis on the correlation between the Beijing family strains and drug resistance also showed that in China, there was an association between Beijing genotype and resistance to RIF, EMB and MDR [21]. In our study, EMB resistance was significantly associated with the Beijing family strains in total samples, and INH and EMB resistance rates were higher in the Beijing genotype strains among adolescents. Further comparison among new cases and previously treated cases also found a strong association between EMB resistance and Beijing strains in previously treated cases in total samples, as well as resistance to RIF, STR, MDR and Beijing strains in previously treated cases of children. Our results are in agreement with the above studies [11,21]. The differences between studies conducted in China may be due to the source of isolates. The Chinese data included in the comprehensive review were from three single studies (Shanghai, Henan and Hong Kong) [19]. Yang et al. enrolled isolates from six field sites located in six different provinces of China [20]. In contrast, the strains of Pang et al. were from nation-wide survey (31 provinces) [11], and our collection was also geographically representative (25 provinces). Finally, the meta-analysis explored publications that dealt with various sources of isolates [21]. Therefore, the results of the latter two studies $[11,21]$ and this study may better represent a real situation.

\section{Conclusion}

In conclusion, this study for the first time analyzed the genetic diversity of $M$. tuberculosis from children in China at the countrywide level; its results were further compared to those obtained on the isolates from adolescents and adults. As a result, the prevalence of Beijing genotype strains was higher in northern and central China, and the lowest in southern China. The genetic characteristics of $M$. tuberculosis isolates were found similar in all three age groups, in particular marked with overall predominance of the Beijing family. The Beijing strains were significantly associated with RIF, STR and MDR resistance in previously treated children.

\section{Acknowledgments}

We thank the staff of the respective institutes and hospitals in different provinces and autonomous regions in China for their excellent contribution to this research.

\section{Author Contributions}

Conceived and designed the experiments: AS KW WJ. Performed the experiments: WJ ZL RH XZ FD HD HH JT QL LL QY WS. Analyzed the data: WJ ZL RH. Contributed reagents/materials/analysis tools: AS KW. Wrote the paper: WJ AS KW.

5. Kremer K, Glynn JR, Lillebaek T, Niemann S, Kurepina NE, et al. (2004) Definition of the Beijing/W lineage of Mycobacterium tuberculosis on the basis of genetic markers. J Clin Microbiol 42: 4040-4049.

6. WHO | Guidelines for surveillance of drug resistance in tuberculosis. WHO. Available: http://www.who.int/tb/publications/2009/surveillance_guidelines/ en/index.html. Accessed 20 Aug 2013.

7. Van Embden JD, Cave MD, Crawford JT, Dale JW, Eisenach KD, et al. (1993) Strain identification of Mycobacterium tuberculosis by DNA fingerprinting: recommendations for a standardized methodology. J Clin Microbiol 31: 406409. 
8. Wan K, Liu J, Hauck Y, Zhang Y, Liu J, et al. (2011) Investigation on Mycobacterium tuberculosis Diversity in China and the Origin of the Beijing Clade. PLoS One 6: e29190.

9. Dong H, Liu Z, Lv B, Zhang Y, Liu J, et al. (2010) Spoligotypes of Mycobacterium tuberculosis from Different Provinces of China. J Clin Microbiol 48: 4102-4106.

10. Guo Y-L, Liu Y, Wang S-M, Li C-Y, Jiang G-L, et al. (2011) Genotyping and drug resistance patterns of Mycobacterium tuberculosis strains in five provinces of China. Int J Tuberc Lung Dis 15: 789-794.

11. Pang Y, Zhou Y, Zhao B, Liu G, Jiang G, et al. (2012) Spoligotyping and Drug Resistance Analysis of Mycobacterium tuberculosis Strains from National Survey in China. PLoS One 7: e32976.

12. Bifani PJ, Mathema B, Kurepina NE, Kreiswirth BN (2002) Global dissemination of the Mycobacterium tuberculosis W-Beijing family strains. Trends Microbiol 10: 45-52.

13. Van Soolingen D, Qian L, de Haas PE, Douglas JT, Traore H, et al. (1995) Predominance of a single genotype of Mycobacterium tuberculosis in countries of east Asia. J Clin Microbiol 33: 3234-3238.

14. Lopez B, Aguilar D, Orozco H, Burger M, Espitia C, et al. (2003) A marked difference in pathogenesis and immune response induced by different Mycobacterium tuberculosis genotypes. Clin Exp Immunol 133: 30-37.
15. Tsenova L, Harbacheuski R, Sung N, Ellison E, Fallows D, et al. (2007) BCG Vaccination Confers Poor Protection Against M. tuberculosis HN878-induced Central Nervous System Disease. Vaccine 25: 5126-5132.

16. Hanekom M, Gey van Pittius NC, McEvoy C, Victor TC, Van Helden PD, et al. (2011) Mycobacterium tuberculosis Beijing genotype: a template for success. Tuberculosis (Edinb.) 91: 510-523.

17. Parwati I, van Crevel R, van Soolingen D (2010) Possible underlying mechanisms for successful emergence of the Mycobacterium tuberculosis Beijing genotype strains. Lancet Infect Dis 10: 103-111.

18. Xing L, Liu R, Li O, Peng Z, Zhu C (2012) Clinical and genotypic characteristics of childhood tuberculosis in Chongqing, China. Eur J Clin Microbiol Infect Dis 31: 1735-1739.

19. European Concerted Action on New Generation Genetic Markers and Techniques for the Epidemiology and Control of Tuberculosis (2006) Beijing/ W Genotype Mycobacterium tuberculosis and Drug Resistance. Emerg Infect Dis 12: 736-743.

20. Yang C, Luo T, Sun G, Oiao K, Sun G, et al. (2012) Mycobacterium tuberculosis Beijing strains favor transmission but not drug resistance in China. Clin Infect Dis 55: 1179-1187.

21. Liu B-B, Lu L-P, Lü B, Wan K-L, Yan Y (2012) [Meta analysis on the correlation between Mycobacterium tuberculosis Beijing family strains and drug resistance]. Zhonghua Yu Fang Yi Xue Za Zhi 46: 158-164. 